\title{
Understanding the product: Backtracking the QFD connections via the product structure
}

\author{
Roger Johansson PhD \\ ${ }^{1}$ Dalarna University, Sweden \\ rjo@du.se
}

\begin{abstract}
The subjectivity involved when creating a Quality Function Deployment (QFD) and the need for companies to understand how their product is fulfilling the customer needs are the focus of this paper. Literature is pointing out the subjectivity when using QFD. Therefore, a method was developed and explored with the aim to try to backtrack and verify the QFD connections via the product structure and thereby reduce the uncertainty. The method calculates a connection strength value for the QFD by summoning connection strength values for three matrices: customer requirements to functions, the functions to technical solutions and technical solutions to product properties. The model was implemented in a relation database and tested using a simple product. The test shows some promising results but also shows sources to uncertainty. Some identified reason for uncertainty were for instance unbalanced matrices, difficult estimates of ratios and redundancy in matrices. The test shows that by working with the model and try to understand why the model gives different results than the QFD gives a better understanding of the product.
\end{abstract}

Keywords: QFD, Product structure, Product development, Customer requirements

\section{Introduction}

It may not be the immediate reaction for a company with a stable increasingly mature product to analyse what exactly in the product takes care of the different customer requirements posed on the product. As competition increases and the sales and revenues start to decrease the motivation to understand what can be done to improve increases significantly. In small and medium sized companies this is a more common scenario. This could in fact be the very first time that they need to, in more detail, understand the products technical solutions' connection to perceived customer values. This translation of the customer's requirements is important for guiding design, verification and process development.(Goderstad \& Haskins, 2016). There is a need for a way that helps the small companies to understand how their product satisfies their customers' needs. This paper aims at proposing a model that might improve and clarify the understanding of the connection between the products measurable properties and the customer requirements. 
In section two only some of the vast amount of related work is presented and an attempt is made to place this research into the context. In section three the research method is briefly explained. Section four describes the proposed method and calculations model used to explore the possibility to verify the connections between customer requirements and product properties. In section 5 the test product is explained. In section 6 a reference QFD is presented. Section 7 explains the experimentation and section 8 and 9 discusses the results and makes some conclusions.

\section{Related work}

There are different tools and methods for the different stages in the product development process. The tools that focus on connecting customer requirements to the products technical solutions are mainly applied in the front-end process(Ulrich \& Eppinger, 2012). Methods used in the front-end process are Functional Decomposition with different approaches(Pahl \& Beitz, 2007; Suh, 1990). Functional decomposition is a tool to use when describing the product in a solution neutral way. It gives a good understanding for what the product should do. The most common method in literature for the connection of customer values to existing product is QFD that, in the first step, tries to connect the customer's ideas of a good quality product to the decisions concerning measurable target values for the product's properties(Mizuno \& Akao, 1994).

There are a vast amount of literature discussing QFD and its application and benefits (Sivasamy et al., 2016) and (Chan \& Wu, 2002) are giving a good picture of this. Efforts are made in different directions to improve certain aspects of QFD. It is combined with e.g. the kano model to identify quality factors, fuzzy logic to better estimate product design time or TRIZ in order to improve innovation(Yeh et al., 2011).

(Sivasamy et al., 2016) are summarizing some of the drawbacks authors have pointed out regarding QFD. One of the drawbacks mentioned is that the developing of the chart is difficult and that the analysis of the data is done in a subjective manner which leads to an inconsistent outcome. "The relationships between WHAT's and HOW's are not accurately indicated ".

(van de Poel, 2007) concludes that customer requirements are product dependent and the correlations between customer requirements and product properties cannot always be treated as non-negative and constant which make the interpretation of the connection challenging.

(Leary \& Burvill, 2007)also discuss the obstacles in QFD and one of them is the correlation between the customer requirements and the product properties which leads to uncertainty when a single technical requirement correlates with multiple customer requirements in both a positive and negative way. They propose an enhancement where the positive and negative correlation can be represented in the QFD matrix.

Attempts to improve the rating of the correlation between customer demands and product properties has been done by changing the traditional scale. (Tacho \& Kwang-Jae, 1998) notes that the conventional rating of relationships primarily shows ordinal relationship ranks between customer requirements and product properties rather than a continuum of rating values that would indicate a more sliding scale of relationship strength. They proposed a cardinal scale instead of a ordinal scale using a swing method. 
(Shabestari \& Bender, 2017) attempts to calculate the relation between customer requirements and product properties using mathematical modeling. To calculate the relations a sensitivity method is used, and the conclusions made by the authors is that there is indication that the methodology is able to calculate the correlations more accurately than the subjectivity of the traditional methods.

The research presented in related work above discusses the correlation between the customer requirements and product properties in three ways: Is there a connection?, if there is a connection how strong is it? and also the characteristic of the correlation i.e. in what way does the connection affect the outcome. Aside from (Shabestari \& Bender, 2017) sensitivity method that relies on a product model there is some subjectivity involved in these decisions. To complement the research this paper discusses and explores an alternative method for connecting in a logical way the existing product 's product properties to the customer values expressed by the customer. and thereby somewhat decreasing the inconsistency and subjectivity in deciding the correlations. The verification proposed is made by evaluating the logical chain suggested by the product development process. The verification aims at verifying if there exist a connection and if it exists is it possible to calculate the strength. The characteristic of the connection as discussed by (Leary \& Burvill, 2007) and (van de Poel, 2007) is not taken into account. Furthermore, the exploration of the method tries to evaluate to some point if the method is good way to help the companies to understand how their product in a technological way provides the value to their customers.

\section{Research method}

The exploration of the proposed method is done through experimentation using a model and was performed in the following steps: 1) a simple product with a reasonable complexity was chosen. 2) a reference QFD was made where the connections between customer requirements and product properties were established the traditional way. 3) the product structure was established and the model for the product was created. 4) Experiments was performed by testing different ways of indicating the correlations in the model and calculate the connection strength figures for the QFD. 5) For each test a comparison was made with the reference QFD to see if the model would indicate similar connections and strength.

\section{The method and assumptions}

The scenario for this research is a company that based on their existing product tries to develop an understanding for how their product fulfils the target values for their product properties. How do they create this understanding? Well, they could do a traditional QFD in the four steps presented by (Mizuno \& Akao, 1994). Marketing will provide the insight of customer requirements and engineers and marketing together tries to decide the measurable product properties. Another approach would be to establish a structure where the customer requirements, functions, technical solutions and measurable product properties were connected. This is referred to as the product structure in this paper.

The engineers could establish a connection matrix between technical solutions (TS) and viable product properties (PP) for the same, a TS_PP matrix. Furthermore, the engineer could establish a connection matrix between the functions (F) for the product and the technical solutions of the product, a F_TS matrix. This is all according to the general concept generation process in product development (Ulrich \& Eppinger, 2012). Now if the engineers and marketing together 
could create a connection matrix between customer requirements (CR) and F, a CR_F matrix, there would be a foundation for backward verification of the traditional QFD, a CR_PP matrix. However, the CR_F matrix is not a common approach and could cause confusion. The thinking behind the above is as follows. There are six possible combinations of the TS, PP, CR, F mentioned above, see Table 1.

Table 1. Six possible combinations of TS, PP, CR and F to create connection matrices.
1. CR_F
2. F_PP
3. CR_PP
4. F_TS
5. CR_TS
6. TS PP

Some of the combinations may not be viable but functions connected to customer requirements CR_F is possible. Function connected to technical solutions F_TS is supported by the product development process in general. Technical solutions connection to product properties TS_PP needs no comment. The CR_TS connection is not easy to do and F_PP could be very difficult to establish. The above reasoning gives two ways to generate this back tracing of the CR_PP matrix, Table 2

Table 2. Two alternatives for back tracing the CR_PP matrix.

1. $\mathrm{TS} P P+\mathrm{F} T \mathrm{TS}+\mathrm{F} \mathrm{CR} \Rightarrow \mathrm{CR} \mathrm{PP}$

2. F_PP + F_ TS $_{-}+\mathrm{CR}_{-}^{-} \mathrm{TS} \Rightarrow \mathrm{CR}_{-}{ }_{-} \mathrm{PP}$

Alternative 1 seems to be the most viable combination and were chosen for further exploration.

The main idea for the backtracking is to sum the connection strength for each of the connections in alternative 1. How to define the connections strength is part of the experimenting and exploration of the method idea. Figure 1, shows a visualisation of the way the sum is calculated.

To calculate the CR_PP connection value for CR3 to PP2, CR3_PP2 the idea was the following.

CR3_PP2 $=((\text { CR_F1+TS_FS3+TS_PP3 })+(\text { CR_F2+TS_F2+TS_PP1 }))^{*}$ CR ranking for CR3

The idea was to see whether this would indicate a high strength for this connection. By comparing the values in the CR_PP matrix one could maybe see weak and strong connections. If this would be the case it might be possible to compare the result with the QFD made in the traditional way to see if the results would verify it. It was also interesting to see if experimentation with the model would indicate other things concerning the products structure.

Based on the idea that it is possible to backtrack the QFD, CR_PP connection matrix a model was built in a relation database. The relations were built based on alternative 1 in Table 2 and the visualisation in Figure 1. It is clear that there is a many to many relations between CR and $F$ i.e. there could be many CR's connected to one F or vice versa. The same is applicable for the connections F to TS and TS to PP. In order to solve this there is a need for a connection table for each of the connections. The connection tables handle the connection strength in the connection matrices. To do the calculation of the CR_PP connection strength as explained earlier it was possible to use a crosstab function. 


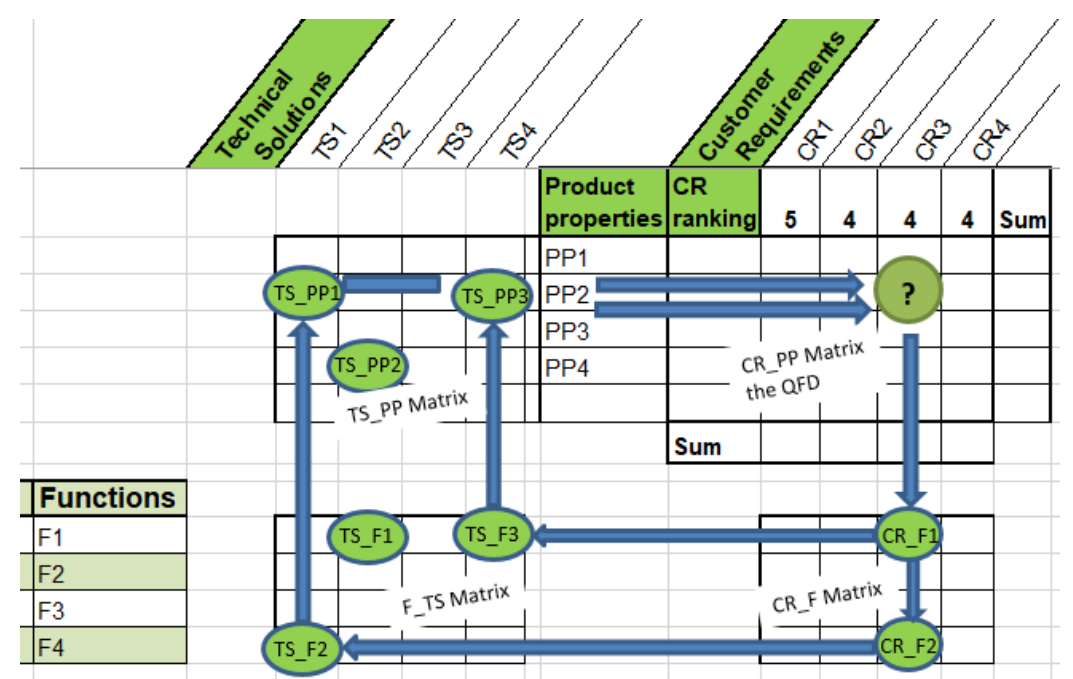

Figure 1. A visualisation on how the backtracking is done for the CR_PP value.

\section{The test product}

In order to test the model and explore the possibilities of backtracking through the product structure a simple product was chosen, the coffee cup. The test product is simple enough to avoid a large complex database but still able to show the model's strengths and weaknesses. The main question is after all: Is it possible to verify the connections between customer requirements and the measurable product properties by following and evaluating the logical connections in the product structure?

\subsection{The customer requirements}

Table 3. Customer Requirements used in the model test with ranking.

\begin{tabular}{|l|c|l|c|}
\hline Easy to drink & 5 & Easy to hold without dropping & 4 \\
\hline Keep the temperature of the coffee & 4 & It should not be too hot to hold & 4 \\
\hline Not easy to turn over & 4 & Fit in the coffee machine & 3 \\
\hline Appealing Design & 3 & Not easy to spill & 3 \\
\hline Should fit in a cupholder & 2 & Easy to clean & 2 \\
\hline Easy to find & 1 & & \\
\hline
\end{tabular}

The customer requirements used for the test product is shown in Table 3 with its ranking on scale 1-5 where rank 5 is the most important requirement.

\subsection{The functions to satisfy the customer requirements}

Table 4. The functions used in the model test.

\begin{tabular}{|l|l|}
\hline Carry information & Accommodate hot liquid \\
\hline Insulate liquid from surrounding environment & Protect the liquid \\
\hline Allow for easy gripping & Prevent tilting \\
\hline Prevent spill & Control the liquids flow direction \\
\hline
\end{tabular}

Table 4, shows the functions used in the model. The functions are means for satisfying the customer requirements. The functions have been discussed and decided as a part of an assignment for the bachelor students in mechanical engineering. If the number of functions is sufficient, correct and if the functions are detailed enough is a question for the model to indicate. 


\subsection{The technical solutions used to carry out the functions.}

Table 5. The technical solutions used in the model test.

\begin{tabular}{|l|l|l|}
\hline Lid & Handle & Spout \\
\hline Vessel & RFID tag & \\
\hline
\end{tabular}

The technical solutions are the realisation of the functions of the product. A question here is how general the technical solutions should be presented. If this model would be used during new product development and a concept is chosen during the concept selection process, for instance according to the method of Concept Screening(Pugh, 1990), the concept may not be more detailed than sparse ideas and make it difficult to use the model. However, in this test the idea and the main focus of the model is to use it to create an understanding of an existing product and verify which product properties are vital for satisfying which customer requirements. Therefore, the selected technical solutions must be sufficient in detail.

\subsection{The product properties to specify the technical solutions}

Table 6. The product properties used in the model test.

\begin{tabular}{|l|l|}
\hline Material & Heat insulation \\
\hline Volume Vessel & Height of vessel \\
\hline Stability & Centre of gravity distance from bottom \\
\hline Form & Weight \\
\hline No of handles & Diameter of spout \\
\hline Surface temperature & Handles placement measured fr bottom \\
\hline Surface texture & Surface smoothness \\
\hline Amount of information & Diameter of vessel \\
\hline
\end{tabular}

The product properties will in detail, with target values, specify the design of the technical solutions. The purpose of the model is to connect the properties to the customer requirements based on the structural thinking of product development team as they go through the development process.

\section{The QFD to verify}

An effort was made to do a QFD that could be used as the reference for the model see Figure 2. The experimentation will be compared with this in order to see if the model is able to verify connections in the QFD. The value "9" is a strong connection and " 3 " is an average connection, weak connections are ignored. 


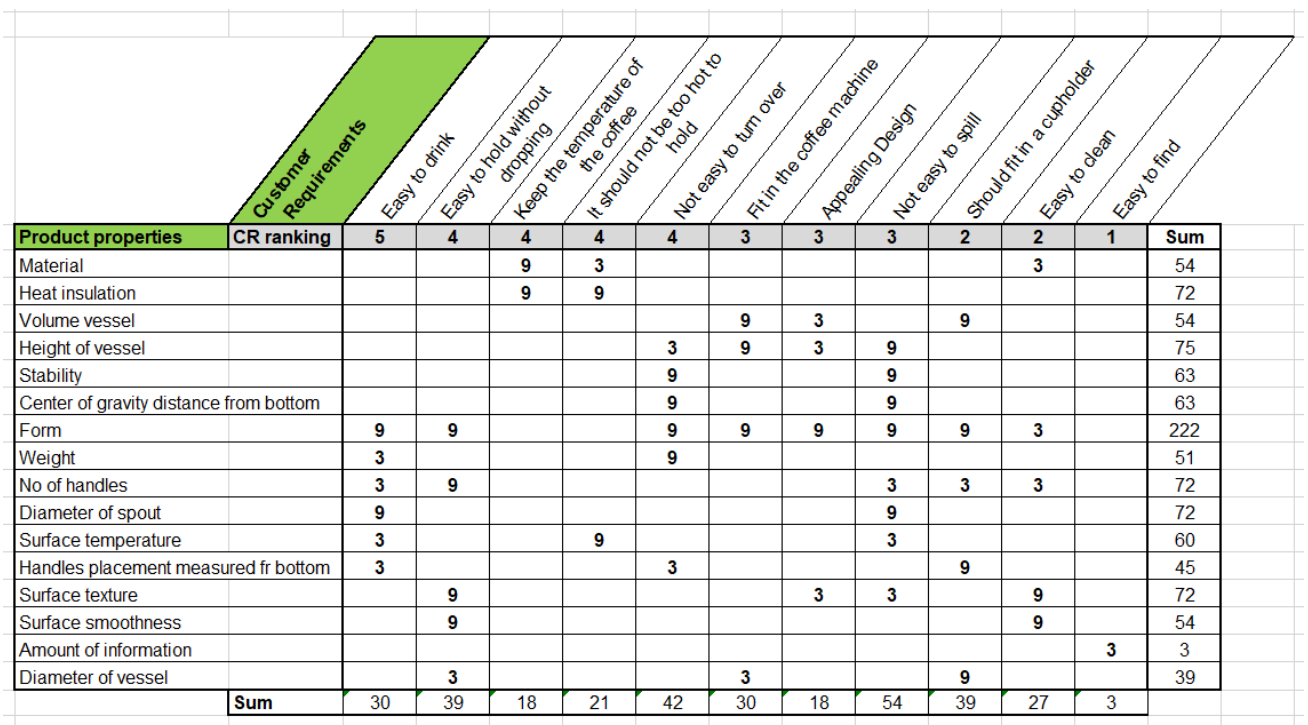

Figure 2. The QFD for the coffee cup used as a reference in the model testing.

\section{Experimentation using the test model}

The experimentation was done in three steps and some of the questions during the simulations were:

- How should the connections in the connection matrices be handled and defined?

- Does the difference in number between CR, F, TS and PP have an effect on how well the CR_PP connection strength verify the QFD?

- What does the CR_PP connection value mean as it is currently defined? See section 4

- How should the CR_PP connection matrix be interpreted in order to understand the strength of the connection?

The first test run was focusing on just indicating in the matrices where there is a connection. A number " 1 " was used to indicate a connection.

In test run number two, a more sophisticated definition of connection strength was used. The connections were defined as ratios. In the CR_F connection matrix the functions share of meeting a certain CR was estimated. For example for fulfilling the CR "appealing design" the F's, "accommodate hot liquid", "allow for easy gripping" and "prevent tilting" is involved. The corresponding estimated share of the F's were 0.7, 0.2 and 0.1 . The same applies for the F_TS connection matrix where a TS share of fulfilling the F is estimated and the TS_PP connection where the TS share of a certain PP is estimated, see Figure 3.

In test run number three, a modification was made for the ratio in the F_TS matrix based on an idea that it is perhaps easier to give the ratio for how much of a function is carried out by the different technical solutions e.g. the $\mathrm{F}$ " insulate liquid from surrounding.." was fulfilled by Lid 0.3 and Vessel 0.7, see Figure 4.

\section{Discussion}

The result from the first test run was not promising as most of the CR_PP matrix was filled with very similar values and there was hard to say anything about the difference in connection strength based on these. 


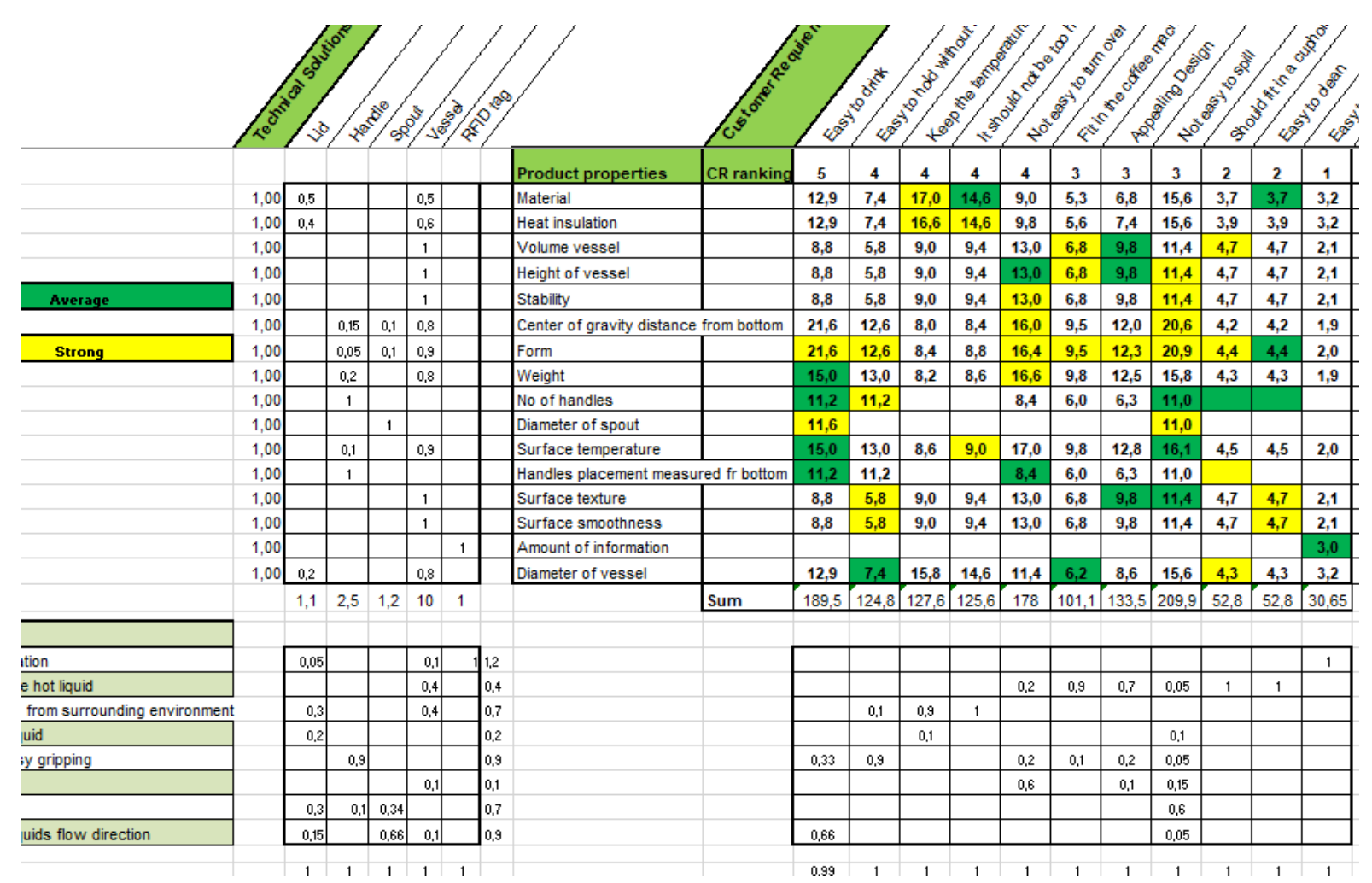

Figure 3 . Result from test run number 2, the colored numbers represent the QFD to be verified.

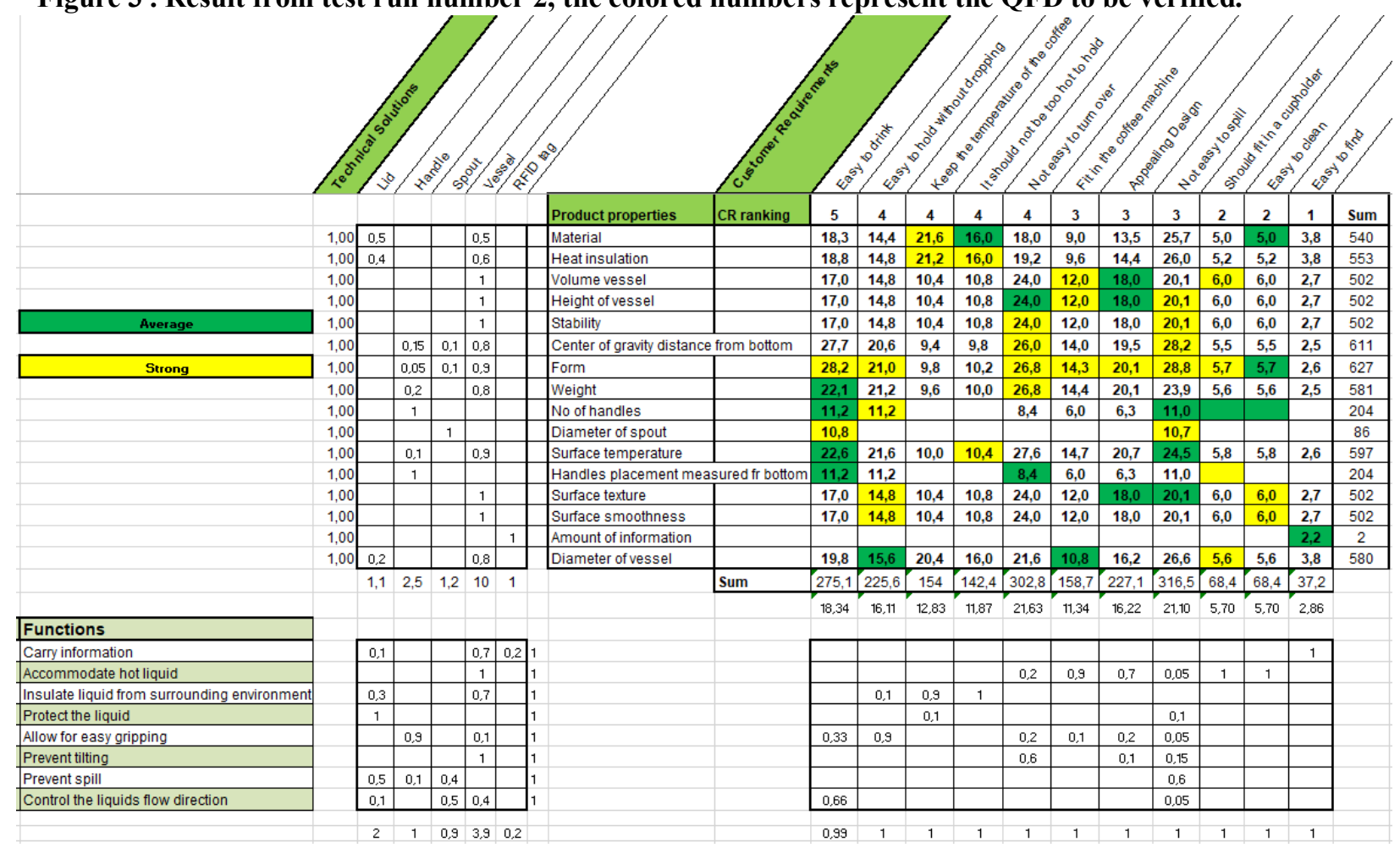

Figure 4. Result from test run number 3. The colored numbers represent the QFD to be verified.

Results from test run two can be seen in Figure 3. The coloured numbers represent the QFD to be verified. As can be seen there are some matches but there are also some large numbers not represented in the QFD. There are even connections in the QFD not represented by the model which can be seen as the coloured cells with no numbers. The difference in results between test run three and four can be seen by examining Figure 3 and Figure 4. 
The tests shows connection strength in a sliding scale more like a continuum of rating values as mentioned by (Tacho \& Kwang-Jae, 1998). The values originate from the product structure and in that sense should be representative. The question is how representative.

The interpretation of the calculated values in the CR_PP matrix can be done in three ways.

1. The values in each column, CR, are compared and the higher values are those with highest connection strength.

2. The values in each row, PP, are compared and the higher values are those with highest connection strength.

3. The values for the whole CR_PP matrix are compared, and intervals are set for each level of connection strength.

The only viable alternative with the definition used in the test is alternative 1 since the connection strength values for CR to PP is then compared with equal customer weight.

Using alternative 1 and evaluating each CR column in the CR_PP matrix shows that the model have the high values in the coloured cells in many of the cases, but sometimes give high rank to other cells than the original QFD has indicated see Figure 4. Some explanations for this are presented below.

The test shows that the definition of the connection strength in the individual matrices makes a big difference on how well the model correlates with the reference QFD. The use of ratio in the connection matrices put subjectivity in the model and the results depends largely on how well these estimates can be done. It is a clear weakness, however compared to the subjectivity when doing the QFD it may be an improvement since the engineers may have more insight when doing these estimates. The connection CR_F is particularly difficult to perform.

One aspect of the model that would cause the results to be inconclusive is an unbalanced model. An unbalanced model could be defined as large difference in number of items in the matrices. For instance, if there would be few F's and many TS's the connection values will be more similar in the CR_PP matrix since many of the CR_PP values share the same route when they are calculated. More items in the matrices would increase the resolution of the calculation and probably give better result. This means that the functional decomposition of the product analysed must be more thoroughly done. This would support the idea of uncoupling as proposed by (Suh, 1990), as the F_TS matrix could be seen as a design matrix. The ratio system used in the test could perhaps be seen as a compensation for the fact that the model is unbalanced.

An explanation for some of the high values in cells that are not set as connections in the original QFD could be the degree of redundancy of some of the CR, F, TS and PP. When deciding the connections in the model the redundancy makes it hard to take a clear decision.

Furthermore, what does the calculated CR_PP value indicate and is it a value that would indicate a strong connection? The value could indicate how many connection paths from CR to PP there are, and it also reflects the strength of the connections on its path. In that way it could be an indication of the importance of the path therefore one could argue that it is a strong connection. The CR_PP value could maybe also indicate the amount information needed for the carrying out that connection in the development of the product which would in a way relate to the information axiom by(Suh, 1990) . 


\section{Conclusions and further work}

This paper has explored the possibility to backtrack and verify the connections in an ordinary QFD via the product structure of customer requirements, functions, technical solution and product properties. A model has been introduced and tested using a simple product. The test shows some promising results but also shows sources to uncertainty. Some identified reason for uncertainty were for instance unbalanced matrices, difficult estimates of ratios and redundancy in matrices. Another question in this work was whether the backtracking of the QFD would give the user of the model a better understanding for the product and how it meets the customer requirements. The test indicates that just by working with the model and try to understand why the model gives different results than the QFD gives a higher degree of understanding.

Would this work with a more complex product? It is hard to say at this moment, more work must be done in order to evaluate the use of the model. Perhaps combine this method with the swing method used by (Tacho \& Kwang-Jae, 1998) or the sensitivity analysis proposed by (Shabestari \& Bender, 2017).

\section{References}

Chan, L.-K., \& Wu, M.-L. (2002). Quality function deployment: A literature review Elsevier European Journal of Operational Research, 143, 463-497.

Goderstad, A. B., \& Haskins, C. (2016). Understanding mission objectives and priorities with QFD. INCOSE International Symposium, 26(1), 283-299. doi:10.1002/j.23345837.2016.00160.x

Leary, M., \& Burvill, C. (2007). Enhancing the Quality Function Deployment Conceptual Design Tool. Journal of Mechanical Design, 129(7), 701-708. doi:10.1115/1.2722787

Mizuno, S., \& Akao, Y. (1994). QFD - the customer-driven approach to Quality Planning and Deployment Asian Productivity Organization.

Pahl, G., \& Beitz, W. (2007). Engineering design -A Systematic Approach (K. Wallace, Trans. L. Blessing Ed. 3 ed.). London: Springer-Verlag.

Pugh, S. (1990). Total design: integrated methods for successful product engineering. Wokingham: Addison-Wesley.

Shabestari, S. S., \& Bender, B. (2017). Enhanced integrated sensitivity analysis in model based QFD method. Paper presented at the the 21st International Conference on Engineering Design (ICED 17) Vol 4: Design Methods and Tools, Vancouver, Canada.

Sivasamy, K., Arumugam, C., Devadasan, S. R., Murugesh, R., \& Thilak, V. M. M. (2016). Advanced models of quality function deployment: a literature review. 50(3), 1399-1414. doi:10.1007/s11135-015-0212-2

Suh, N. P. (1990). The Principles of Design. New York: Oxford University Press New York.

Tacho, P., \& Kwang-Jae, K. (1998). Determination of an optimal set of design requirements using house of quality. Journal of Operations Management, 16(5), 569-581.

Ulrich, K. T., \& Eppinger, S. D. (2012). Product design and development. (Vol. 5): Boston : McGraw-Hill.

van de Poel, I. (2007). Methodological problems in QFD and directions for future development. Research in Engineering Design, 18(1), 21-36. doi:10.1007/s00163-007-0029-7

Yeh, C. H., C., J., Huang, Y., \& Wu, F. C. (2011, June 26-29). A Breakthrough Product R\&D Model by Using the integration of Four-phases QFD's and TRIZ. Paper presented at the Modelling, Identification and Control, Shanghai, China. 